\title{
INTERMEDIATE ENERGY SOLAR NEUTRINOS
}

\author{
E. Calabresu ${ }^{a}$, G. Fiorentini ${ }^{\mathrm{b}}$, M. Lissia $^{\mathrm{a}}$ and B. Ricci ${ }^{\mathrm{c}}$ \\ âDipartimento di Fisica dell'Università di Cagliari, I-09124 Cagliari \\ and Istituto Nazionale di Fisica Nucleare, Sezione di Cagliari, I-09127 Cagliari, Italy \\ ${ }^{\text {b}}$ Dipartimento di Fisica dell'Università di Ferrara, I-44100 Ferrara \\ and Istituto Nazionale di Fisica Nucleare, Sezione di Ferrara, I-44100 Ferrara, Italy \\ ${ }^{\mathrm{c}}$ Dipartimento di Fisica dell'Università di Padova, I-35100 Padova \\ and Istituto Nazionale di Fisica Nucleare, Sezione di Ferrara, I-44100 Ferrara, Italy
}

\begin{abstract}
We extract information on the fluxes of Be and CNO neutrinos directly from solar neutrino experiments, with minimal assumptions about solar models. Next we compare these results with solar models, both standard and non standard ones. Finally we discuss the expectations for Borexino, both in the case of standard and non standard neutrinos.
\end{abstract}

\section{Introduction}

The principal aim of this paper is to extract information on the fluxes of Be and CNO neutrinos directly from solar neutrino experiments, with minimal assumptions about solar models. In this respect, we will update previous results 值司 and try to elucidate the role of CNO neutrinos. We will see that experimental data are more and more against the hypothesis of standard neutrinos (i.e. without mass, mixing, magnetic moments...).

Next we will compare these informations with solar models, both standard and non standard ones. Clearly, low (i.e. smaller than standard) central temperature models are ruled out, essentially because they cannot reproduce the experimental data available on both $\mathrm{Be}$ and $\mathrm{B}$ neutrinos. Hybrid models, where some suitable nuclear cross section is varied in order to reduce the Be neutrinos flux to the observed value and with a higher central temperature, so as to agree with experimental results on $\mathrm{B}$ neutrinos flux, can also be excluded, as in these models the CNO neutrino flux grows beyond acceptable levels. In other words, the bounds on Be (CNO) neutrinos tell us that it is hopeless to reduce (enhance) the central solar temperature, in order to stay with standard neutrinos.

In summary, we shall demonstrate that, under the assumption of standard neutrinos:

- the available experimental results look inconsistent among themselves, even if one of the four experiments were wrong;

- the flux of intermediate energy neutrinos $(\mathrm{Be}+\mathrm{CNO})$ as derived from experiments is significantly smaller than the prediction of SSM's;

- the different reduction factors for ${ }^{7} \mathrm{Be}$ and ${ }^{8} \mathrm{~B}$ neutrinos with respect to the SSM are essentially in contradiction with the fact that both ${ }^{7} \mathrm{Be}$ and ${ }^{8} \mathrm{~B}$ neutrinos originate from the same parent ${ }^{7} \mathrm{Be}$ nucleus.

We will discuss then the expectations for Borexino, both in the case of standard and non standard neutrinos, showing that the experiment can clearly discriminate among several possible solutions to the solar neutrino puzzle.

\section{Where are Be and CNO neutrinos?}

We make the assumption of stationary Sun (i.e. the presently observed luminosity equals the present nuclear energy production rate) and standard neutrinos, so that all the $\nu_{e}$ produced in the Sun reach Earth without being lost and their energy spectrum is unchanged. The relevant vari- 
Table 1

For the $i$-th neutrino flux, we show the average neutrino energy $\langle E\rangle_{i}$ and the energy averaged capture cross sections in Chlorine $\left(\sigma_{i, C}\right)$ and Gallium $\left(\sigma_{i, G}\right)$. Errors correspond to 1 standard deviation and

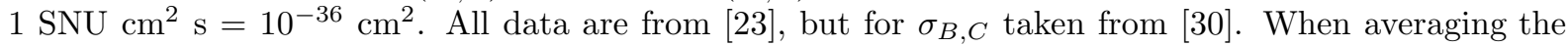
pp and pep components to get $\mathrm{p}$, we use the relative weights of the SSM from Ref. [15]; similarly for ${ }^{13} \mathrm{~N}$ and ${ }^{15} \mathrm{O}$ to get $\mathrm{CNO}$.

\begin{tabular}{|c|c|c|c|}
\hline & $\begin{array}{c}\langle E\rangle_{i} \\
{[\mathrm{MeV}]}\end{array}$ & 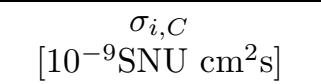 & $\begin{array}{c}\sigma_{i, G} \\
{\left[10^{-9} \mathrm{SNU}^{2} \mathrm{~cm}^{2} \mathrm{~s}\right]}\end{array}$ \\
\hline $\mathrm{pp}$ & 0.265 & 0. & $(1 \pm 0.02)$ \\
\hline pep & 1.442 & $(1 \pm 0.02)$ & $(1 \pm 0.07)$ \\
\hline $\mathrm{p}=\mathrm{pp}+\mathrm{pep}$ & 0.268 & $0.38 \cdot 10^{-2}(1 \pm 0.02)$ & $(1 \pm 0.02)$ \\
\hline${ }^{7} \mathrm{Be}$ & 0.814 & $(1 \pm 0.02)$ & $(1 \pm 0.03)$ \\
\hline${ }^{13} \mathrm{~N}$ & 0.707 & $(1 \pm 0.02)$ & $(1 \pm 0.03)$ \\
\hline${ }^{15} \mathrm{O}$ & 0.996 & $(1 \pm 0.02)$ & $(1 \pm 0.06)$ \\
\hline $\mathrm{CNO}={ }^{13} \mathrm{~N}+{ }^{15} \mathrm{O}$ & 0.842 & $(1 \pm 0.02)$ & $(1 \pm 0.05)$ \\
\hline${ }^{8} \mathrm{~B}$ & 6.71 & $1.11 \cdot 10^{3} \quad(1 \pm 0.03)$ & $2.43 \cdot 10^{3}(1 \pm 0.25)$ \\
\hline
\end{tabular}

ables are thus the (energy integrated) neutrino fluxes, which can be grouped as:

$\Phi_{\mathrm{pp}+\mathrm{pep}}, \quad \Phi_{\mathrm{Be}}, \quad \Phi_{\mathrm{CNO}}$ and $\Phi_{\mathrm{B}}$.

These four variables, see [2,3], are constrained by four relationships:

(a) the luminosity equation, which tells that the fusion of four protons (and two electrons) into one $\alpha$ particle is accompanied by the emission of two neutrinos, whichever is the cycle:

$K_{\odot}=\sum_{i}\left(\frac{Q}{2}-\langle E\rangle_{i}\right) \Phi_{i}$

where $K_{\odot}$ is the solar constant $\left(K_{\odot}=8.533 \cdot 10^{11}\right.$ $\left.\mathrm{MeV} \mathrm{cm}{ }^{-2} \mathrm{~s}^{-1}\right), \mathrm{Q}=26.73 \mathrm{MeV}$ and $\langle E\rangle_{i}$ is the average energy of the $\mathrm{i}$-th neutrinos.

(b) The Gallium signal $S_{G}=(74 \pm 8)$ SNU (weighted average between the Gallex 6] and SAGE [7] results) can be expressed as a linear combination of the $\Phi_{i}$ 's, the weighting factors $\sigma_{i, G}$ being the absorption cross section for the ith neutrinos, averaged on their energy spectrum, see Table 1 for updated values:

$S_{G}=\sum_{i} \sigma_{i, G} \Phi_{i}$ (c) A similar equation holds for the Chlorine experiment, $S_{C}=(2.55 \pm 0.25) \mathrm{SNU}$ [8]:

$S_{C}=\sum_{i} \sigma_{i, C} \Phi_{i}$

(d) The Kamiokande experiment determines - for standard neutrinos - the flux of Boron neutrinos [9]:

$\Phi_{\mathrm{B}}=(2.73 \pm 0.38) \cdot 10^{6} \mathrm{~cm}^{-2} \mathrm{~s}^{-1}$.

With the numerical values in Table 11, from Eq. (2) (after dividing both term by $Q / 2$ ) and Eqs. (3) and (4) one gets:

$$
\begin{aligned}
63.85 & =0.980 \Phi_{\mathrm{pp}+\mathrm{pep}}+0.939 \Phi_{\mathrm{Be}} \\
& +0.937 \Phi_{\mathrm{CNO}}+0.498 \cdot 10^{-3} \Phi_{\mathrm{B}} \\
74 \pm 8 & =1.23 \Phi_{\mathrm{pp}+\mathrm{pep}}+7.32 \Phi_{\mathrm{Be}} \\
& +8.72 \Phi_{\mathrm{CNO}}+2.43 \Phi_{\mathrm{B}} \\
2.55 \pm 0.25 & =0.38 \cdot 10^{-2} \Phi_{\mathrm{pp}+\mathrm{pep}}+0.24 \Phi_{\mathrm{Be}} \\
& +0.41 \Phi_{\mathrm{CNO}}+1.11 \Phi_{\mathrm{B}},
\end{aligned}
$$

where all fluxes are in units of $10^{9} \mathrm{~cm}^{-2} \mathrm{~s}^{-1}$, but the B flux which is in units of $10^{6} \mathrm{~cm}^{-2} \mathrm{~s}^{-1}$. Only errors on experimental signals are kept, since, to a first approximation, they are dominant for determining fluxes. 
The three equations (2), (3) and (4) together with (5) imply a unique solution:

$$
\begin{aligned}
\Phi_{\mathrm{Be}} & =(0.4 \pm 6.6) \cdot 10^{9} \mathrm{~cm}^{-2} \mathrm{~s}^{-1} \\
\Phi_{\mathrm{CNO}} & =(-2.0 \pm 4.8) \cdot 10^{9} \mathrm{~cm}^{-2} \mathrm{~s}^{-1} \\
\Phi_{\mathrm{pp}+\text { pep }} & =(66.7 \pm 2.0) \cdot 10^{9} \mathrm{~cm}^{-2} \mathrm{~s}^{-1}
\end{aligned}
$$

One notes that the central value for $\Phi_{\mathrm{CNO}}$ is unphysically negative. At first sight, this seems not to be a problem, in view of the estimated error. However, there is a strong correlation between the errors.

In order to understand what is going on, and to make clear the role of each experimental result, let us reduce the number of equations and of unknowns by the following tricks:

(a) one can eliminate $\Phi_{\mathrm{pp}+\text { pep }}$ by using the luminosity equation (2);

(b) since $\langle E\rangle_{C N O} \geq\langle E\rangle_{B e}$, the corresponding cross section has to be larger than that of Be neutrinos. Thus the minimal CNO signal is obtained with the replacement

$\sigma_{C N O} \rightarrow \sigma_{B e}$

(We remark that this is also a safe approach, since the theoretical value of $\sigma_{B e, G}$ has essentially been verified to the $10 \%$ level by the Gallex neutrino source experiment [10]).

In this way, the above equations can be written in terms of two variables, $\Phi_{\mathrm{Be}+\mathrm{CNO}}$ and $\Phi_{\mathrm{B}}$, and the results of each experiment can be plotted in the $\left(\Phi_{\mathrm{B}}, \Phi_{\mathrm{Be}+\mathrm{CNO}}\right)$ plane, see Fig. 囵.

Clearly all four experiments point towards $\Phi_{\mathrm{Be}+\mathrm{CNO}}<0$. This means that the statement "neutrinos are standard and experiments are correct" has lead us to an unphysical conclusion. Could the problem be with some experiment? It is clear from Fig. 1 that the situation is unchanged by arbitrarily disregarding one of the experiments, see Ref. 11].

As an attempt of being more quantitative, by applying standard statistical arguments to Eqs. (6) we can derive the following conclusions: (1) the chance $P$ for the unknown variable $\Phi_{\mathrm{Be}+\mathrm{CNO}}$ to be positive is less than about $2 \%$. Should we disregard arbitrarily one of the experiments, still one has $P \leq 6 \%, 7 \%$ or $8 \%$ neglecting respectively the results of Chlorine, Gallium or
Kamiokande. This indicates that standard neutrinos $\left(\Phi_{\mathrm{Be}+\mathrm{CNO}} \geq 0\right)$ are unlikely.

(2) To the $99.5 \%$ C.L., the unknown variable $\Phi_{\mathrm{Be}+\mathrm{CNO}}$ should not exceed $0.7 \cdot 10^{9} \mathrm{~cm}^{-2} \mathrm{~s}^{-1}$.

(3) To the same confidence level, if one assumes a priori standard neutrinos (and therefore $\Phi_{\mathrm{Be}+\mathrm{CNO}} \geq 0$ ) the combined flux of Be and CNO neutrinos does not exceed $2 \cdot 10^{9} \mathrm{~cm}^{-2} \mathrm{~s}^{-1}$.

(4) Similar statements hold for Be flux (take $\Phi_{\mathrm{CNO}}=0$ ) and CNO flux (put $\Phi_{\mathrm{Be}}=0$ in Eqs. (6)), see Table 2.

The main message can be roughly summarized by saying that the chances of standard neutrinos are low, not much more than $2 \%$. However, some caution is needed, since the experimental errors we are using are combinations of statistical fluctuations and systematic uncertainties.

\section{Experimental results and standard solar models}

Let us insist on the hypothesis of standard neutrinos and compare experimental information with theoretical estimates.

We have reported in Fig. 1 the results of several recent solar model calculations (diamonds) $12-19$ together with experimental results. Some of the models predict a $\mathrm{B}$ flux close to the Kamiokande value; however no model is capable of reproducing the low $\mathrm{Be}+\mathrm{CNO}$ flux implied by the experiments.

In Table 2, we have considered only standard solar models where He and heavier element diffusion is taken into account 13, 15, 19], as these should be more accurate. Indeed, the comparison with helioseismology tells us that diffusion is important for solar models to predict the correct depth of the convective envelope 21,22]. We also note that in models with diffusion the central solar temperature is increased: as Helium falls towards the centre, the mean molecular weight increases in the stellar core and a higher temperature is needed to balance the gravitational force. Models with diffusion yield thus even larger Be, $\mathrm{CNO}$ and B neutrinos fluxes.

For standard neutrinos, the experimental information is presented in columns a) and c) of Table 2. The discrepancy between theory and 
Table 2

Information on Be, CNO and B neutrino fluxes. Be and CNO (B) fluxes are in units of $10^{9} \mathrm{~cm}^{-2} \mathrm{~s}^{-1}$ $\left(10^{6} \mathrm{~cm}^{-2} \mathrm{~s}^{-1}\right)$. All bounds are at the $99.5 \%$ C.L. Direct information (a) is only available for B neutrinos, from Ref. [9]. The bounds in (b) correspond to no prior knowledge on the unknown variables. In (c) we assume a priori $\Phi_{i} \geq 0$. The results of SSMs with diffusion are also shown: P94 from Ref. [13], BP95 from Ref. [15], FRANEC95 indicates our preliminary results [19].

\begin{tabular}{lcccccc}
\hline flux & (a) & (b) & (c) & P94 & BP95 & FRANEC95 \\
\hline $\mathrm{B}$ & $2.73 \pm 1.14$ & & & 6.48 & 6.62 & 6.9 \\
$\mathrm{Be}+\mathrm{CNO}$ & & $\leq 0.6$ & $\leq 1.9$ & 6.38 & 6.31 & 6.5 \\
$\mathrm{Be}$ & & $\leq 0.6$ & $\leq 1.9$ & 5.18 & 5.15 & 5.3 \\
$\mathrm{CNO}$ & & $\leq 0.4$ & $\leq 1.4$ & 1.20 & 1.16 & 1.2 \\
\hline
\end{tabular}

experiment is about a factor two for the Boron flux. More important looks to us the discrepancy on $\Phi_{\mathrm{Be}+\mathrm{CNO}}$, where the predicted values exceed the experimental upper bounds by a factor three, at least.

The problem is mostly with beryllium neutrinos and let us examine it in some detail. The extraction of $\Phi_{\mathrm{Be}}$ from experimental data (with the requirement $\Phi_{\mathrm{CNO}} \geq 0$ ) yields an unphysically negative Be flux. Without any prior knowledge, $\Phi_{\mathrm{Be}}$ cannot exceed $1 / 10$ of the SSM prediction at the $99.5 \%$ C.L. If we a priori force it to be non negative, the upper bound is $1 / 5$ of the SSM at the $95 \%$ C.L.; a value as high as $1 / 3$ of the SSM prediction is only allowed at the $99.5 \%$ C.L. All this indicates that Be neutrino suppression is much stronger than that of B neutrinos.

\section{The relevance of Beryllium}

As well known, theoretical predictions are more robust for Be than for B neutrinos, the reasons being the weaker sensitivity to the central solar temperature $T$ and the independence on the (poorly known) astrophysical factor $S_{17}$ for the $\mathrm{p}+{ }^{7} \mathrm{Be} \rightarrow{ }^{8} \mathrm{~B}+\gamma$ reaction. Approximately, one has:

$$
\begin{aligned}
\Phi_{\mathrm{Be}} & =\Phi_{\mathrm{Be}, 0}\left(T / T_{0}\right)^{10} \\
\Phi_{\mathrm{B}} & =\Phi_{\mathrm{B}, 0}\left(S_{17} / S_{17,0}\right)\left(T / T_{0}\right)^{20},
\end{aligned}
$$

where the subscript 0 refers here and in the following to the SSM predictions. For the power law coefficients see [2, 23, 24].

In addition, we point out a relationship between $\Phi_{\mathrm{Be}}$ and $\Phi_{\mathrm{B}}$ which elucidates physically the problem of the relative abundances of $\mathrm{Be}$ and
$\mathrm{B}$ neutrinos. Both Be and B-neutrinos are sons of the ${ }^{7}$ Be nucleus, see Fig. 2. For this nucleus, electron capture (rate $\lambda_{e}$ ) is clearly favoured over proton capture (rate $\lambda_{p}$ ), due to the absence of the Coulomb barrier (it is curious that a weak process has a larger chance than an electromagnetic process, but this is the case due to the exponentially small penetration probabilities of the Coulomb barrier, at the energies of interest to us). Thus the value of $\Phi_{\mathrm{Be}}$ is a clear indicator of the central density $n_{7}$ of the progenitors ${ }^{7}$ Be nuclei:

$n_{7} \propto \Phi_{\mathrm{Be}} / \lambda_{e}$

If $\Phi_{\mathrm{Be}}$ comes out to be reduced by some (large) factor with respect to the SSM prediction, the same holds for the ${ }^{7} \mathrm{Be}$ equilibrium abundance (we recall that $\lambda_{e}$ is weakly dependent on temperature, and it is essentially known from measurements in the laboratory, see Ref. [25]). The puzzle is thus with B neutrinos, since:

$\Phi_{\mathrm{B}} \propto n_{7} \lambda_{p}$

The observed (Kamiokande) value of $\Phi_{\mathrm{B}}$ being just a factor two below the SSM prediction, it looks that experiments are observing too high $\Phi_{\mathrm{B}}$ ! Put it in another way, one cannot kill the father/mother before the baby is conceived.

Should we insist on this road, we need to enhance $\lambda_{p} / \lambda_{e}$. We remark that any attempt to reduce $S_{17}$ goes into the wrong direction.

\section{Reduced central temperature models?}

Non standard solar models with smaller central temperaure can be obtained by varying - well be- 


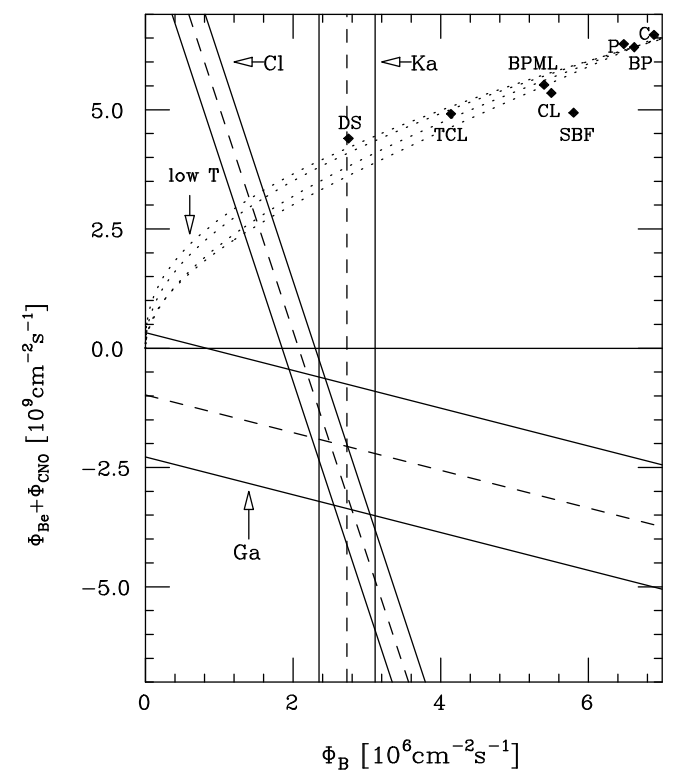

Figure 1. Neutrino fluxes allowed by the present experimental results. Dashed lines correspond to central values of the experimental results, solid lines denote $\pm 1 \sigma$ limits. Diamonds represent recent solar model calculation 12 19. The dotted area corresponds to (non-standard) low temperature solar models [2,20].

yond the estimated uncertainties - a few parameters (the cross section of the pp reaction, chemical composition, opacity, age... [2,20]). These models span the dotted area in Fig. 1, which can be clearly understood by simple considerations.

To a rough approximation, also $\Phi_{\mathrm{CNO}}$ has a power law dependence on temperature [2,23,24]:

$\Phi_{\mathrm{CNO}}=\Phi_{\mathrm{CNO}, 0}\left(T / T_{0}\right)^{20}$

One can use this equation together with Eqs. (9) above; by expressing the temperature as a function of $\Phi_{\mathrm{B}}$, one has:

$$
\begin{aligned}
\Phi_{\mathrm{Be}}+\Phi_{\mathrm{CNO}} & =\Phi_{\mathrm{Be}, 0}\left(\Phi_{\mathrm{B}} / \Phi_{\mathrm{B}, 0}\right)^{1 / 2} \\
& +\Phi_{\mathrm{CNO}, 0}\left(\Phi_{\mathrm{B}} / \Phi_{\mathrm{B}, 0}\right)
\end{aligned}
$$

and one sees in Fig. 1 1 the square root behaviour at small $\Phi_{\mathrm{B}}$, which then changes to linear for larger $\Phi_{\mathrm{B}}$.

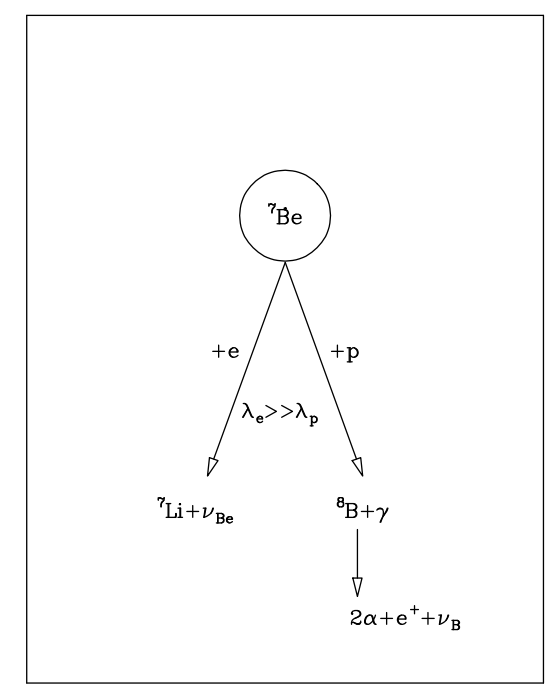

Figure 2. The fate of ${ }^{7} \mathrm{Be}$ nuclei.

It is clear that all these model fail to reproduce the experimental results, essentially because they cannot reproduce the observed ratio $\Phi_{\mathrm{Be}} / \Phi_{\mathrm{B}}$, see Ref. [26], as a consequence of the drastically different dependences on temperature, see Eqs. (9). If $\Phi_{\mathrm{B}}$ is reduced by a factor two, $\Phi_{\mathrm{Be}}$ is too high. On the other hand, if $\Phi_{\mathrm{Be}}$ is brought to the low level required by the experiments, the predicted $\Phi_{\mathrm{B}}$ is definitely too small. In other words, as we said previously, we are observing too many B-neutrinos (if neutrinos are standard)!

\section{Higher central temperatures? (Or why do we care about CNO neutrinos)}

One could imagine the conspiracy of two mechanisms, so as to bring both $\Phi_{\mathrm{Be}}$ and $\Phi_{\mathrm{B}}$ in agreement with experiment. For example, one could assume that $S_{33}$ is much larger than commonly assumed (e.g. as a result of a hypothetical resonance [27) so as to enhance the ppI channel and reduce $\Phi_{\mathrm{Be}}$ to the desired value. At the same time, by varying some suitable parameter the central temperature could be increased, so as to bring $\Phi_{\mathrm{B}}$ in agreement with experiment. 
Table 3

Predictions for Beryllium neutrinos. For different models we present at the best fit point $\left(\sin ^{2} 2 \theta, \delta m^{2}\right)$, the $\chi^{2}$ for degree of freedom, the flux and signal $(\mathrm{CC}+\mathrm{NC})$ in units of the SSM predictions.

\begin{tabular}{|c|c|c|c|c|c|c|}
\hline & & $\chi^{2} /$ d.o.f. & $\sin ^{2} 2 \theta$ & $\delta m^{2}\left[\mathrm{eV}^{2}\right]$ & $\Phi / \Phi_{0}$ & $\mathrm{~S} / \mathrm{S}_{0}$ \\
\hline \multirow[t]{4}{*}{ (a) } & Active neutrinos: & & & & & \\
\hline & MSW small $\theta$ & $0.9 / 2$ & 0.0058 & $7.9 \cdot 10^{-6}$ & $9 \%$ & $27 \%$ \\
\hline & MSW large $\theta$ & $1.5 / 2$ & 0.63 & $1.7 \cdot 10^{-5}$ & $48 \%$ & $59 \%$ \\
\hline & Just-So & $1.9 / 2$ & 1.00 & $6.0 \cdot 10^{-11}$ & $78 \%$ & $82 \%$ \\
\hline \multirow[t]{4}{*}{ (b) } & Sterile neutrinos: & & & & & \\
\hline & MSW small $\theta$ & $0.7 / 2$ & 0.0079 & $4.9 \cdot 10^{-6}$ & $2 \%$ & $2 \%$ \\
\hline & MSW large $\theta$ & $8.1 / 2$ & 0.73 & $1.3 \cdot 10^{-5}$ & $46 \%$ & $46 \%$ \\
\hline & Just-So & $7.2 / 2$ & 0.86 & $6.2 \cdot 10^{-11}$ & $33 \%$ & $33 \%$ \\
\hline
\end{tabular}

This mechanism also fails 28, see Fig. 3, due to the fact that as temperature raises, the $\mathrm{CNO}$ flux grows as fast as the Boron flux, and the experimental bound on $\Phi_{\mathrm{Be}+\mathrm{CNO}}$ is again violated.

In other words, while Beryllium and Boron neutrinos tell us that one cannot hope to solve the neutrino problem by lowering the central temperature, the bound on $\mathrm{CNO}$ implies that increasing the temperature does not work either.

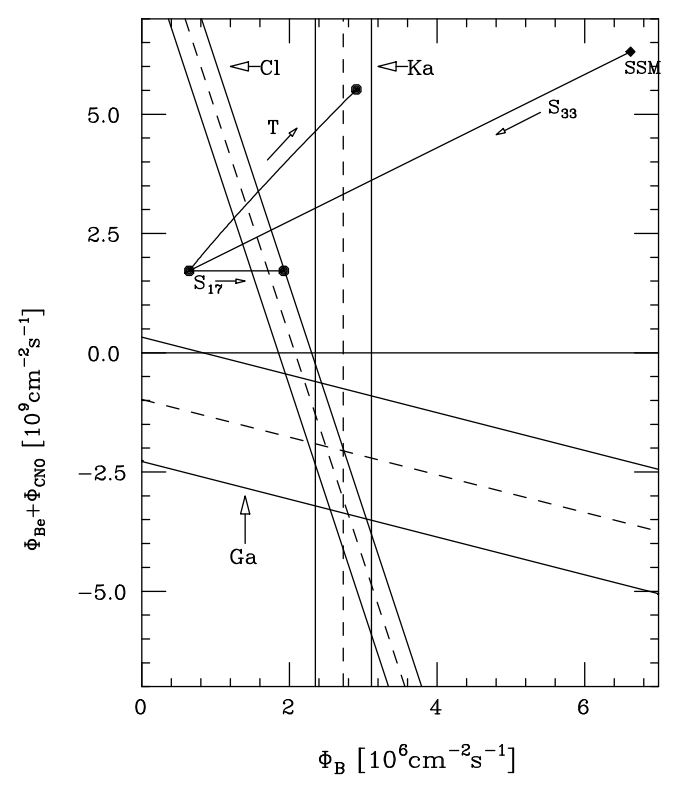

Figure 3. Sketch of the behaviour of solar models with non standard $S_{33}$, central temperature T and $S_{17}$, from Ref. [28].
If instead the temperature is unchanged but $S_{17}$ is increased, one has still the problem that the SSM prediction for the CNO exceeds the experimental constraint, see again Fig. 3 and Ref. [28].

\section{Expectations for Be neutrinos}

We have seen that, for standard neutrinos, the Be-flux is strongly suppressed with respect to the SSM predictions. What has to be expected for non standard neutrinos?

In Table 3 we update and extend a recent analysis 29] for a few candidate solutions. We use now as a reference the fluxes corresponding to the "best model with Helium and metal diffusion" of Ref. 15. For active neutrinos, both small and large angle MSW solutions are acceptable, as well as the Just-So model. On the other hand, for sterile neutrinos only MSW at small angle gives a good fit. Among these four acceptable solutions, two of them (MSW large angle and Just-So) give signals $(\mathrm{CC}+\mathrm{NC})$ that are quite a significant fraction of the SSM prediction, see last column in Table 3. In other words, in face of the present experimental data, the Beryllium signal does not need to be small, for non standard neutrinos.

The situation is made more clear in Fig. Q, where we show the $90 \%$ C.L. regions according to the different models. A direct measurement of the Be line can in many cases discriminate among the possibile solutions. Very large signals, above $75 \%$ of the SSM prediction, correspond essentially to the Just-So solution. Between $75 \%$ and about $35 \%$ various models are acceptable. Between $35 \%$ 
and $20 \%$ the solution has to bee MSW at small angle for active neutrinos. Very small signal, say below $20 \%$, are only possible for standard neutrinos, or transitions into sterile neutrinos.

In the intermediate region discrimination between Just-So and MSW solutions should be obtained by Borexino looking at seasonal variations, even for purities well below the design purity, see [29.

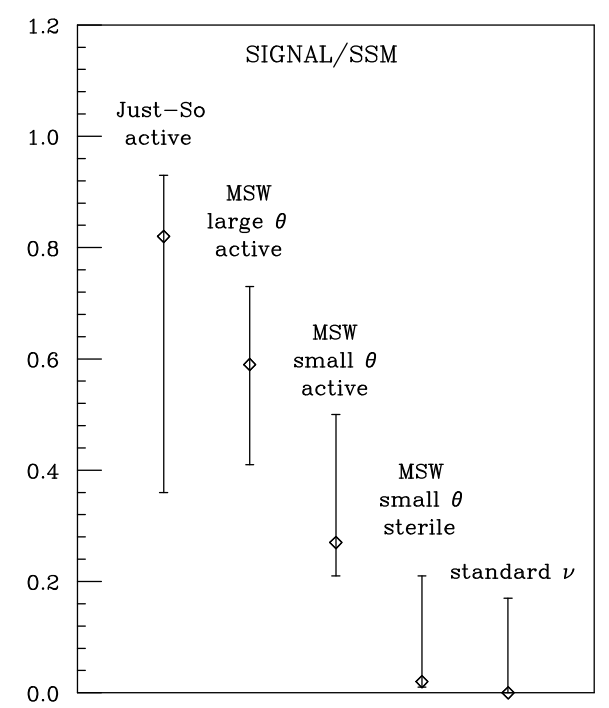

Figure 4. The Beryllium $(\mathrm{CC}+\mathrm{NC})$ signal, in units of the SSM prediction. Diamonds indicate the best fit points, bars correspond to $90 \%$ C.L.

Most of the results presented here are the outcome of the fruitful and friendly collaboration in the last few years with V. Berezinsky, V. Castellani, F. Ciacio, S. Degl'Innocenti and N. Ferrari. We are grateful to T. E. O. Ericson and to G. Cocconi for useful discussions. One of us (G. F.) thanks the CERN Theory Division for hospitality while this work was done.

\section{REFERENCES}

1. V. Castellani, S. Degl'Innocenti and G. Fiorentini, Astron. Astrophys. 271 (1993) 601.

2. V. Castellani, S. Degl'Innocenti, G. Fiorentini, M. Lissia and B. Ricci, Phys. Rev. D 50 (1994) 4749.

3. S. Degl'Innocenti, G. Fiorentini and M. Lissia, Nucl. Phys. B Proc. Suppl. 43 (1995) 67.

4. N. Hata, University of Pennsylvania Report No. UPR-0612T, to appear in: Proc. of the Solar Modeling Workshop (Institute of $\mathrm{Nu}-$ clear Theory, University of Washington, Seattle, March 1994).

5. N. Hata, S. A. Bludman and P. Langacker, Phys. Rev. D 49 (1994) 3622.

6. GALLEX Collaboration, P. Anselmann et al., Phys. Lett. B 357 (1995) 237.

7. SAGE collaboration, J. N. Abdurashitiv et al., Nucl. Phys. B Proc. Suppl. 38 (1995) 60.

8. B. T. Cleveland et.al., Nucl. Phys. B Proc. Suppl. 38 (1995) 47.

9. T. Kajita, ICRR-Report, 332-94-27 (December 1994).

10. GALLEX Collaboration, P. Anselmann, Phys. Lett. B 342 (1995) 440.

11. J. N. Bahcall, Phy. Lett. B 338 (1994) 276.

12. A. Dar \& G. Shaviv, physics e-print archive astro-ph/9401043 (1994).

13. C. R. Proffitt, Ap. J. 425 (1994) 849.

14. S. Turck-Chièze \& I. Lopes, Ap. J. 408 (1993) 347.

15. J. N. Bahcall \& M. H. Pinsonneault, Rev. Mod. Phys., to appear (1995).

16. G. Berthomieu, J. Provost, P. Morel and Y. Lebreton, Astr. Astroph. 268 (1993) 775.

17. I. J. Sackmann, A. J. Boothroyd and W. A. Fowler, Ap. J. 360 (1990) 727.

18. C. Charbonnel and Y. Lebreton, Astr. Astroph. 380 (1993) 666.

19. F. Ciacio et.al., 1995, in preparation.

20. V. Castellani, S. Degl'Innocenti, G. Fiorentini, M. Lissia and B. Ricci, Phys. Lett. B 324 (1994) 425.

21. J. Christensen-Dalsgaard, D. O. Gough and M. J. Thompson, Ap. J. 378 (1991) 413.

22. J. Christensen-Dalsgaard, C. R. Proffitt and 
M. J. Thompson, Ap. J 403 (1993) L75.

23. J. N. Bahcall, Neutrino Astrophysics (Cambridge University Press, Cambridge, 1989).

24. J. N. Bahcall and A. Ulmer, preprint, October 1995.

25. C. Rolfs and R. Rodney "Cauldrons in the Cosmos", The University of Chicago Press, Chicago, 1988.

26. V. Berezinsky, Comm. Nucl. Part. Phys. 21 (1994) 249.

27. W. A. Fowler, Nature 238 (1972) 24.

28. V. Berezinsky, G. Fiorentini and M. Lissia, physics e-print archive astro-ph/9509116, to appear in Phys. Lett. B (1995).

29. E. Calabresu, N. Ferrari, G. Fiorentini and M. Lissia, physics e-print archive hep$\mathrm{ph} / 9507352$, to appear in Astroparticle Physics (1995).

30. M. Aufderheide et.al., Phys. Rev C 49 (1994) 678. 